\title{
IMPROVING GEOMETRIC HASHING BY MEANS OF FEATURE DESCRIPTORS
}

\author{
Federico Tombari and Luigi Di Stefano \\ DEIS-ARCES, University of Bologna, Bologna, Italy \\ \{federico.tombari, luigi.distefano\}@unibo.it
}

Keywords: Geometric hashing, Feature matching, Object recognition.

\begin{abstract}
Geometric Hashing is a well-known technique for object recognition. This paper proposes a novel method aimed at improving the performance of Geometric Hashing in terms of robustness toward occlusion and clutter. To this purpose, it employs feature descriptors to notably decrease the amount of false positives that generally arise under these conditions. An additional advantage of the proposed technique with respect to the original method is the reduction of the computation requirements, which becomes significant with increasing number of features.
\end{abstract}

\section{INTRODUCTION AND PREVIOUS WORK}

Geometric Hashing (GH) (Lamdan and Wolfson, 1988 ) is a powerful and popular technique for object recognition. GH relies on interest points, or features (e.g. corners, edge points, ...) to detect the presence of a particular object, or shape, in an image. $\mathrm{GH}$ is divided into two main stages: Modeling (offline) and Recognition (online) stage. During the first, given the object to be detected, a set of features is extracted from a model image. Then, for each feature pair $\left(x_{1}\right.$, $x_{2}$ ), or basis, all features are transformed to a new coordinate systems where one axis goes through $x_{1}$ and $x_{2}$, and the unit length is the distance between $x_{1}$ and $x_{2}$. For each basis, all feature coordinates are stored into a quantized histogram, or Hash Table. During the Recognition stage, features are first extracted from the image under analysis (hereinafter also referred to as target image). Then, for a feature pair, all the features of the target image undergo the same transformation as done for the model image, so that each transformed coordinate pair casts a vote for the bases that were accumulated in the hash table bin they fall into. If a basis gets a number of votes higher than a pre-defined threshold, then that basis identifies the presence of the object model into the target image, otherwise another feature pair is taken under evaluation. This approach detects objects under similarities (i.e. translation, rotation and scaling), while to deal with affine transformations each basis has to be formed by a feature triplet instead of a pair (Lamdan and Wolfson, 1988).
To above-described method can be detect the presence of multiple object models in the target image, i.e. by considering (model,bases) pairs in both the online and offline stages.

Later studies (Grimson and Huttenlocher, 1990; Lamdan and Wolfson, 1991) have highlighted that GH is particularly sensitive to the presence of clutter and occlusions. In particular, in presence of many spurious features originating from a cluttered background, the necessary use of a high number of bases (which must be taken proportional to the number of possible feature pairs) can easily cause false positives, i.e. false matches due to consistent accumulation of a high number of erroneous votes (this phenomenon is also known as data collision (Chum and Matas, 2006)). This issue is worsened by the presence of occlusions. In fact, even though in principle $\mathrm{GH}$ can deal with partial occlusions (Lamdan and Wolfson, 1988), in practice when too many object features are occluded the few visible ones cannot accumulate enough evidence to grant detection (Simon and Meddah, 2006), especially in presence of data collisions due to clutter. Accurate probabilistic analysis of the error induced by GH can be found in (Grimson and Huttenlocher, 1990; Lamdan and Wolfson, 1991). Another disadvantage of the use of GH is the computational time and memory requirements, which do not scale well with the number of features (Iwamura et al., 2007). As for the former, GH has a computational complexity of $O\left(n^{3}\right)$ (worst case), $n$ being the number of extracted features from the target image (Lamdan and Wolfson, 1988). 

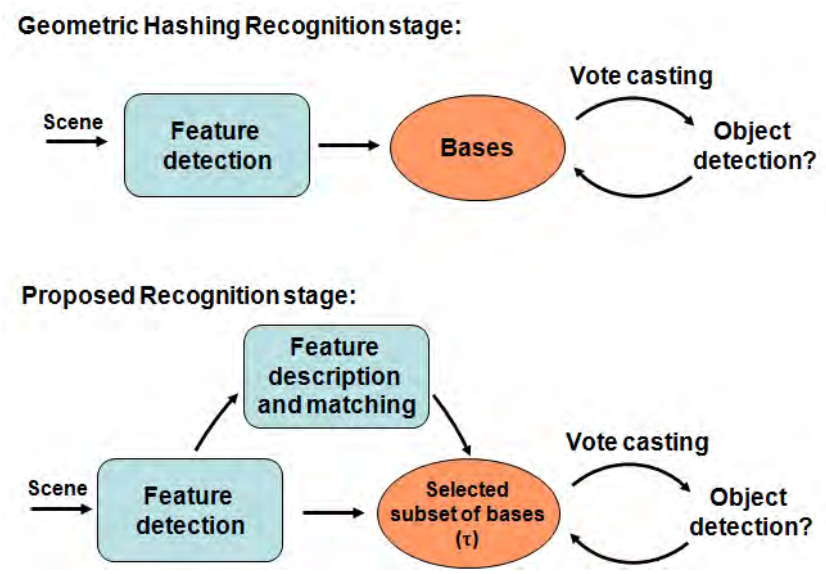

Figure 1: Recognition stage in GH and proposed method.

Several approaches have been proposed in literature to improve the basic GH formulation. (Tsai, 1996) aims at achieving higher robustness to noise using line features instead of interest points. Yet, this approach does not solve the problem of false positives arising in presence of clutter and occlusions. Other approaches aim at reducing the computational cost of $\mathrm{GH}$, e.g. by randomly select the feature points in order to reduce their number (Iwamura et al., 2007) or by limiting the matching domain to a local neighborhood of a feature by means of local invariants (Iwamura et al., 2007). The problem of clutter is explicitly handled in (Sehgal and Desai, 2003) for 3D object recognition by incorporating into the object model the information about each feature position with regards to the object centroid. Then, at Recognition, possible centroid positions casted by GH votes are clustered using k-means. Drawbacks of this approach are represented by an increased computational complexity (of the order of $O\left(n^{4}\right)$ plus the cost of clustering).

In this paper we propose a novel approach that is more robust than $\mathrm{GH}$ to occlusion and clutter as well as more efficient when the number of features and/or models becomes significant. Both aspects are in our opinion extremely important from an application perspective, on one hand because the occlusions and clutter are issues found in many real working conditions, on the other because most industrial applications impose real-time or near-real-time requirements. In particular. the proposed approach deploys feature descriptors to discard those features that are prone to lead to data collisions in the hash table, thus potentially decreasing the number of false positives in presence of occlusions and/or clutter. This also speedups the method since it notably reduces the number of bases that need to be examined during the recognition stage.

\section{GEOMETRIC HASHING WITH FEATURE DESCRIPTORS}

In the last years, a very fertile computer vision research topic has dealt with the so called invariant local features (e.g. (Mikolajczyk et al., 2005; Mikolajczyk and Schmid, 2005; Lowe, 2004; Bay et al., 2008)). In such a framework, image features are detected and described invariantly to specific set of transformations, i.e. similarities or also affinities. In particular, the description stage aims at embedding into a vector distinctive information concerning the local neighborhood of the interest point for the purpose of robustly matching features between images. Such an approach has proved to be successful in many hard computer vision task such as image retrieval, image registration and stitching, camera pose estimation, 3D reconstruction and also object recognition within feature based methods such as Hough voting and RANSAC.

In the GH algorithm, the object model is given by the spatial information represented by the feature locations. We aim at improving the performance of $\mathrm{GH}$ by adding to this representation the distinctive information given by the description of each feature. More precisely, the use of this additional piece of information allows for computing correspondences between the model features and the visible (i.e. notoccluded) object features in the target image, so as to detect those feature points that do not match any model feature and hence are likely to belong to clutter. Accordingly, these cluttering features are not allowed to form possible bases, thus rendering the overall algorithm significantly less prone to possible false positives arising by accidental accumulation of erroneous votes, for erroneous votes that would be casted by feature points when evaluating such bases are indeed no longer casted. An additional advantage of this 
approach is that, since the majority of possible basis choices are discarded, the Recognition stage is significantly faster than with the standard GH algorithm.

We outline here the details of the proposed method. For the sake of clarity and conciseness, we address the case of $2 \mathrm{D}$ object recognition under similarities, though the method can be generalized straightforwardly to deal with affinities as well as for 3D object recognition (Lamdan and Wolfson, 1988; Grimson and Huttenlocher, 1990).

As sketched in Fig. 1, the offline stage is analogous to that of the GH algorithm: i.e. for each model, feature points are detected, then for each feature pair the feature positions are transformed according to the current basis and stored in the Hash Table. In addition, though, we also compute a descriptor for each feature point and store it for later use. Also, we do not consider as possible bases those feature pairs whose distance is either too big or too small: in the former case transformed feature coordinates would get small values and become too prone to noise, in the latter case the Hash Table would become too big and sparse due to transformed feature coordinates getting large values.

As for the online (Recognition) stage, given a model to be sought for, first features are detected and described into the target image. Then, correspondences between features are established by matching each target image descriptor to the model descriptors using as matching measure the euclidean distance. In particular, for each target image descriptor the ratio between the most similar model descriptor and the second-most similar model descriptor is computed. This nearest-neighbor search can be efficiently implemented using efficient indexing techniques such as $K d$-trees (Beis and Lowe, 1997). Once this is done for all target image features, they are sorted in increasing order of match confidence based on this ratio: obviously, the smaller the ratio value, the higher the probability that the current feature belongs to the model. Then, only the first $\tau$ features are selected to form possible bases: all the other features are discarded and won't be considered as possible bases. The size of this subset of features, $\tau$, is a parameter of the algorithm: in our experiments we have empirically selected the value of 10 . Hence the number of features used to generate bases, $n_{b}$, is given by:

$$
n_{b}=\min (\tau, n)
$$

Given this subset of features, $S_{n_{b}}$, in turn each feature pair is selected from $S_{n_{b}}$ as the current basis. Also in this case, we adopt the approach of not considering feature pair whose distance is either too big or too small. Once a basis is selected, all the other features extracted (not just those belonging to $S_{n_{b}}$ ) are transformed according to the current basis and used for casting votes as in the original $\mathrm{GH}$ algorithm. If votes are accumulated in one (or more) bin of the Hash table, then the current object is found, otherwise another basis is evaluated until either the object is detected or all bases have been evaluated.

It is worth pointing out that the proposed approach can easily deal with the presence of multiple object instances into the target image by evaluating all overthreshold bins in the Hash Table obtained with a particular basis. As for the computational burden, it is important to note that although our method requires additional computations in the Recognition stage in order to describe and match interest points, efficient algorithms do exist for both tasks (Lowe, 2004; Bay et al., 2008)). Moreover, our method notably speeds up the "vote casting" process, so that the complexity of the Recognition stage, which is $O\left(n^{3}\right)$ in the standard algorithm, is reduced to $O\left(\tau^{2} n\right)$, where $\tau$ (i.e. the number of features which are allowed to generate bases) can easily be one order of magnitude smaller than $n$. Hence, complexity is linear in the number of features instead of cubic: this also allows for the use of a high number of features which, as it will be shown in the next section, helps improving the performance of the algorithm.

\section{EXPERIMENTAL RESULTS}

This section presents an experimental evaluation where the proposed approach is compared to the standard GH algorithm in an object recognition scenario. In particular, we propose two different experiments based on two different datasets.

\subsection{Experiment 1}

In Experiment 1, an object has to be recognized within a test dataset composed of 40 images. The test dataset is characterized by object translations, rotations and -quite large- scale changes. Moreover, there is a strong presence of clutter and occlusions. In each of the 40 test images the object to be recognized always appears once. The object model and a few test images are shown in Fig.2. Correct matches are determined by evaluating the position error between the ground-truth bounding box around the object and that found by the algorithm. More specifically, we compare the performance of the two algorithms by means of Recall vs. Precision curves, by varying the threshold applied on the peaks of the Hash table. To compute the Recall and Precision terms, a True Positive 


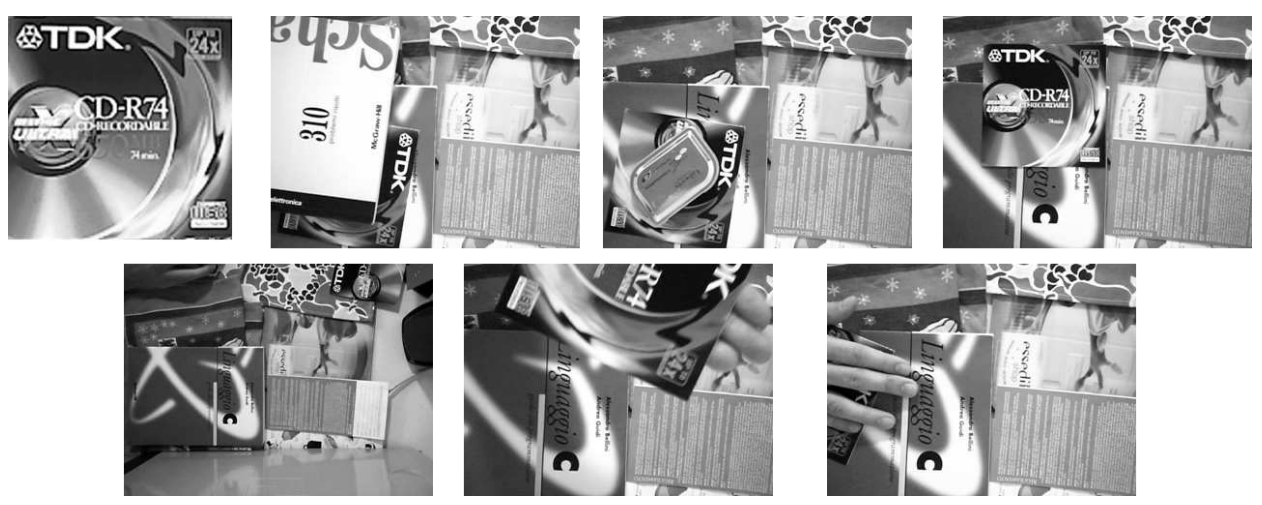

Figure 2: A subset of the dataset used in Experiment 1 (top left: the object model).
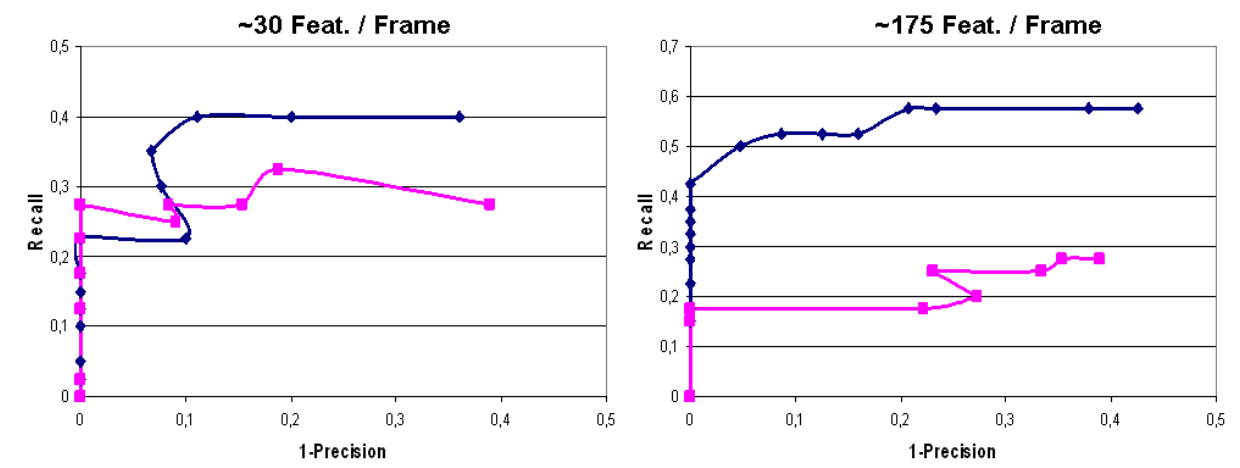

BEST

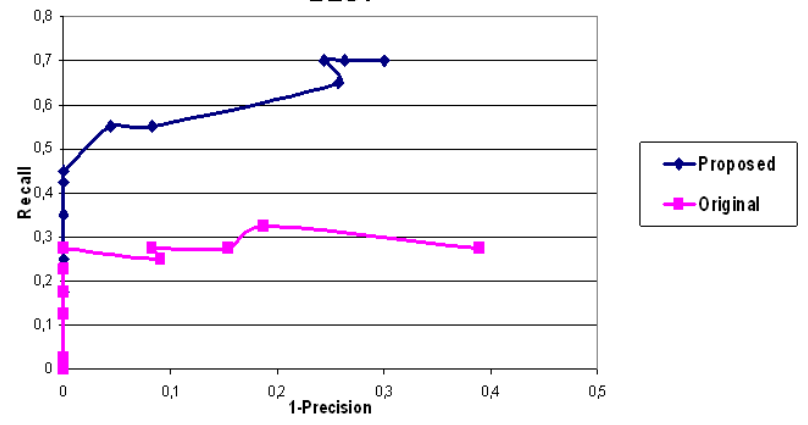

Figure 3: Experiment 1: comparison between GH and the proposed algorithm with different amounts of extracted features.

(TP) occurrs only when the 4 coordinates pairs of the corners of the object found by the object recognition algorithm are close enough to the ground-truth ones (ground-truth was obtained by hand-labeling).

As for parameters, The Hash Table quantization parameter has been tuned on the dataset, and has the same value for both algorithms. As for the feature descriptor, we have selected the well-known SIFT descriptor (Lowe, 2004), which has been shown to be discriminative and efficient (Mikolajczyk and Schmid, 2005). Interest points for both GH and the proposed approach are extracted by means of the DOG detector (Lowe, 2004), which is known to be highly repeatable under several disturbance factors such as, e.g., viewpoint changes, noise, illumination changes, blur (Mikolajczyk et al., 2005).

We provide experimental results varying the number of extracted SIFT features. The resulting curves are shown in Fig. 3. The left chart on top shows the result using a small number of features - 30 on the average per frame (the same features are extracted and used with both methods). Instead, the right chart on top concerns a higher number of features (175 on the average per frame, same features for both methods). As it can be seen, the proposed approach notably outperforms the original GH method in both experiments, typically yielding a higher Recall at equal (1-Precision). It is also interesting to note that the 

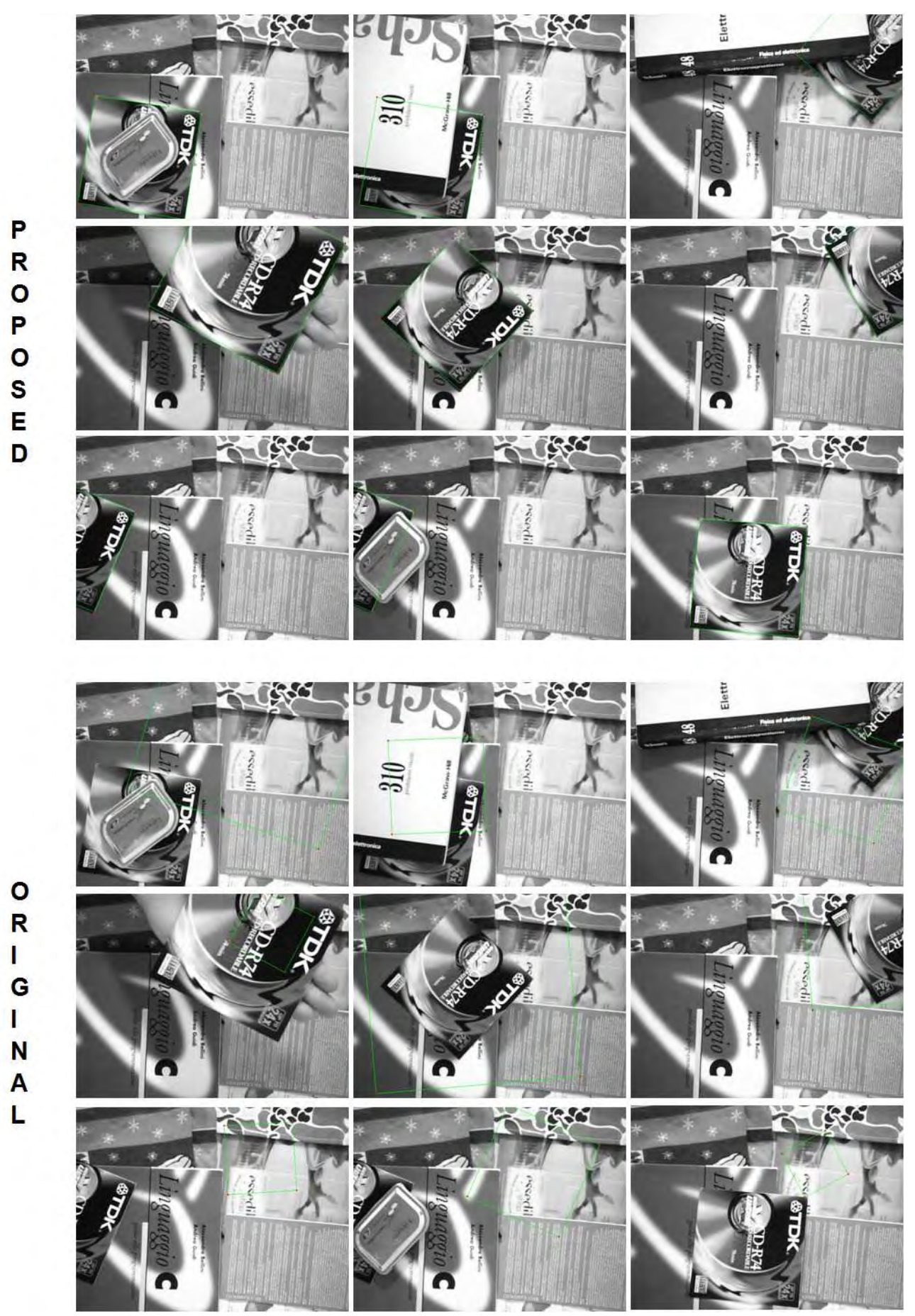

Figure 5: Experiment 1: detection results (shown by a green bounding box) yielded by the proposed algorithm (top) and GH (bottom) on a subset (9 images) of the evaluated dataset.

original GH method obtains better performance in presence of a few number of features, mainly due to the arousal of false positives when the feature number increases. On the contrary, and as expected, the proposed method can benefit of a higher number of features since the use of a small and fixed number of "good" bases prevents from the malicious effect of coherent accumulations of erroneous votes. In addition, the chart on the bottom shows the performance of the two algorithms using the best number of features for 


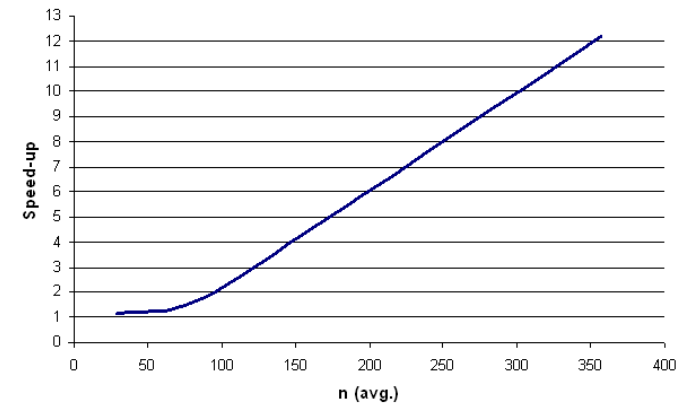

Figure 4: Speed-up of proposed method over GH.

each method: as it can be seen, the proposed method clearly outperforms the GH algorithm.

In addition, we provide indications on the efficiency of both algorithms by showing, in Fig. 4, the measured speed-ups of the proposed algorithm with regards to GH at different numbers of extracted features: the proposed method is always faster than $\mathrm{GH}$, the speed-up increasing significantly as $n$ gets higher.

Finally, in Fig. 5 we provide qualitative results concerning the detection capabilities of both algorithms. More specifically, the Figure shows, by means of a green bounding box, the output of the object detection on a subset of the dataset composed of 9 images. As it can be seen from the Figure, and as it was already indicated by the quantitative results shown in Fig. 3, the proposed algorithm allows for a notably more robust object detection with respect to $\mathrm{GH}$ in presence of clutter and occlusions.

\subsection{Experiment 2}

As for Experiment 2, we deploy a larger dataset with respect to that of Experiment 1 that is composed of 3 object models and 500 test images. This dataset concerns an industrial application aiming at recognizing specific magazines. More specifically, 30 images of the test dataset contain one instance of the object models (10 images for each model), while the remaining 470 images do not contain any object model instance. This dataset includes the presence of rotations and translations of the objects, in addition there is the presence of clutter (due to the presence of other magazines) and occlusions (ranging between 5-20\%). A subset of this dataset is shown in Fig. 6.

The determination of correct matches is accomplished exactly in the same way as in Experiment 1. Also for this experiment, the same feature detector, descriptor and Hash Table parameter value used for Experiment 1 were deployed. Moreover, we use the same parameter values of the SIFT features for both methods so to extract the same features from each image. Due to the bigger dimensions of the test images of this dataset compared to the ones used in Experiment 1 , and to the fact that on the average these images are more textured, by means of the same parameter values used in the previous experiments we get a much higher number of features (on the average, $\sim 1200$ features are extracted from each test image).

The resulting Precision-Recall curves are shown in Fig. 7. As it can be seen, also here the proposed approach yields notable benefits in terms of recognition accuracy compared to the original $\mathrm{GH}$ formulation.

\section{CONCLUSIONS}

We have proposed a novel method for object recognition that improves the performance of the GH algorithm in presence of clutter and occlusions. The use of similarity information between features, obtained through the use of feature descriptors, helps selecting a subset of good features that can reliably used in the GH framework for object recognition in the current scene, while filtering out those creating clutter. An additional benefit brought in by the proposed approach is the much higher computational efficiency when the number of interest points is high.

\section{REFERENCES}

Bay, H., Ess, A., Tuytelaars, T., and Gool, L. V. (2008). Surf: Speeded up robust features. Computer Vision and Image Understanding, 110(3):346-359.

Beis, J. and Lowe, D. (1997). Shape indexing using approximate nearest-neighbour search in high dimensional spaces. In Proc. CVPR, pages 1000-1006.

Chum, O. and Matas, J. (2006). Geometric hashing with local affine frames. In Proc. IEEE Conf. on Computer Vision and Pattern Recognition, pages 879-884.

Grimson, W. and Huttenlocher, D. (1990). On the sensitivity of geometric hashing. In Proc. Int. Conf. on Computer Vision, pages 334-338.

Iwamura, M., Nakai, T., and Kise, K. (2007). Improvement of retrieval speed and required amount of memory for geometric hashing by combining local invariants. In Proc. BMVC2007, pages 1010-1019.

Lamdan, Y. and Wolfson, H. (1991). On the error analysis of 'geometric hashing'. In Proc. IEEE Conf. on Computer Vision and Pattern Recognition, pages 22-27.

Lamdan, Y. and Wolfson, H. J. (1988). Geometric hashing: A general and efficient model-based recognition scheme. In Proc. ICCV, pages 238-249.

Lowe, D. G. (2004). Distinctive image features from scaleinvariant keypoints. International Journal of Computer Vision, 60:91-110. 

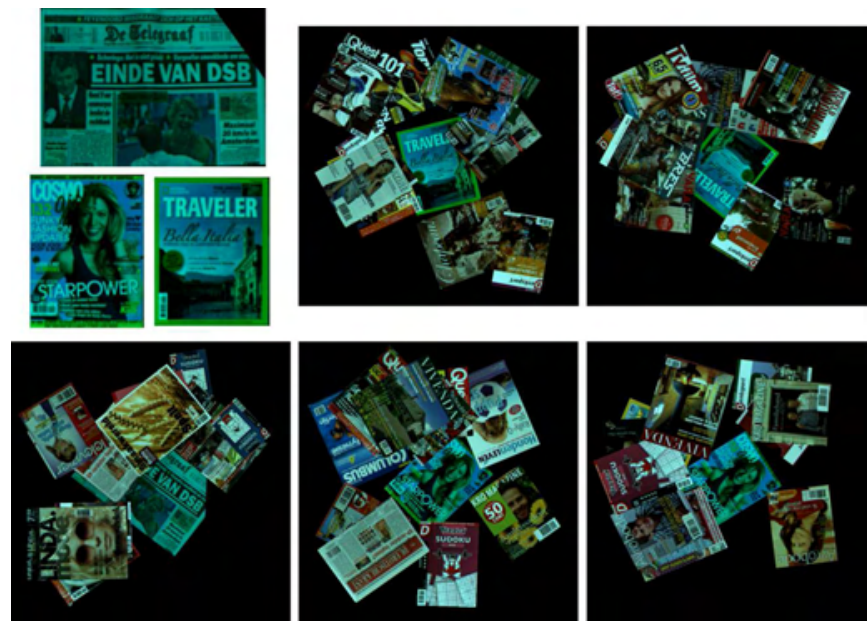

Figure 6: Dataset 2: the 3 object models (top left) and 5 examples of the test images (each one showing one instance of an object model).

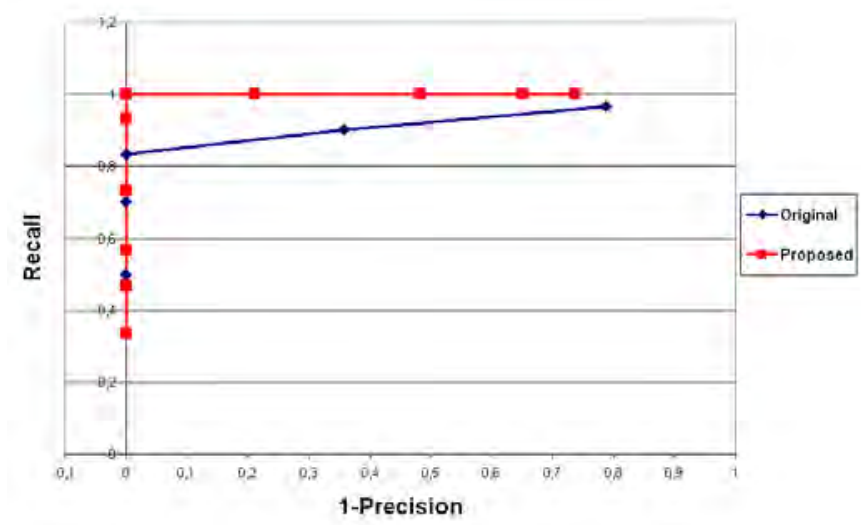

Figure 7: Comparison between $\mathrm{GH}$ and the proposed algorithm on the dataset of Experiment 2.

Mikolajczyk, K. and Schmid, C. (2005). A performance evaluation of local descriptors. PAMI, 27(10):16151630.

Mikolajczyk, K., Tuytelaars, T., Schmid, C., Zisserman, A., Matas, J., Schaffalitzky, F., Kadir, T., and Gool, L. V. (2005). A comparison of affine region detectors. Int. J. Comput. Vision, 65(1-2):43-72.

Sehgal, A. and Desai, U. (2003). 3d object recognition using bayesian geometric hashing and pose clustering*1. Pattern Recognition, 36(3):765-780.

Simon, C. and Meddah, D. (2006). Geometric hashing method for model-based recognition of an object. US Patent 7027651.

Tsai, F. (1996). A probabilistic approach to geometric hashing using line features. Computer Vision and Image Understanding, 63(1):182-195. 